\begin{tabular}{|c|l|}
\hline Title & $\begin{array}{l}\text { Stereoselective synthesis of 4-fluoro-1,3-3l kadieny lboronates and their application in the stereoselective synthesis of } \\
\text { fluoropolyenes }\end{array}$ \\
\hline Author(s) & Sakai, Miho; Guan, Tong; Hara, Shoji \\
\hline Citation & $\begin{array}{l}\text { Journal of Fluorine Chemistry, 128(12), 1444.1448 } \\
\text { https://doi.org/10.1016/.jluchem.2007.07.009 }\end{array}$ \\
\hline Issue Date & $2007-12$ \\
\hline Doc URL & http://hdl.handle.net/2115/30291 \\
\hline Type & article (author version) \\
\hline File Information & JFC128 12.pdf \\
\hline
\end{tabular}

Instructions for use 


\title{
Stereoselective synthesis of 4-fluoro-1,3-alkadienylboronates and their application in the stereoselective synthesis of fluoropolyenes
}

\author{
Miho Sakai, Tong Guan, Shoji Hara* \\ Division of Chemical Process Engineering, Graduate School of Engineering, \\ Hokkaido University, Sapporo 060-8628, Japan
}

\begin{abstract}
E, 3E)- and (1E, 3Z)-4-Fluoro-1,3-alkadienylboronates were stereoselectively prepared by the Heck-reaction of a vinylboronate with $(E)$ - or (Z)-2-fluoro-1-alkenyliodonium salts, and applied to the Suzuki-Miyaura coupling reaction for the synthesis of fluoropolyenes.
\end{abstract}

Keywords: Heck-reaction, Fluoroalkadienylboronates, Suzuki-Miyaura coupling, Fluoropolyene, Fluoroalkenyliodonium salts

\section{Introduction}

Polyene natural products that exhibit significant bioactivities are widely known in nature $[1,2]$. The fluorinated analogs of the polyene are of great interest for studying their actions in vivo and the modification of their activities [3-8]. Fluorodienylmetals (1) are effective as building blocks for the synthesis of polyene analogs with fluorine attached to the double bonds $[5,8]$. Although fluorodienylboronates $(\mathbf{1}, \mathrm{M}=\mathrm{B}$ in scheme 1) can be used as building blocks for the synthesis of various fluoropolyenes by the Suzuki-Miyaura coupling reaction [9], they have not yet been synthesized.

*Corresponding author. Fax: +81-11-706-6556

e-mail address: shara@ eng.hokudai.ac.jp 


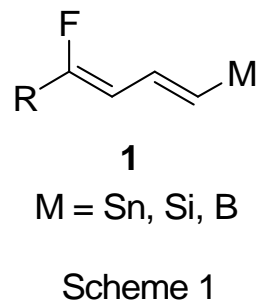

Alkadienylboronates are synthesized by the Heck reaction of vinylboronates (4) with haloalkenes and used for polyene synthesis [10, 11]. Recently, we succeeded in the stereoselective synthesis of (E)- and (Z)-2-fluoro-1-iodoalkenes (2) and 2-fluoroalkenyliodonium salts (3) [12-14]. For the synthesis of fluoroalkadienylboronates (5) and their application in the synthesis of fluoropolyenes (6), we examined the Heck reaction of vinylboronates (4) with 2-fluoro-1-iodoalkenes 2 and 2-fluoroalkenyliodonium salts 3 (Scheme 2).

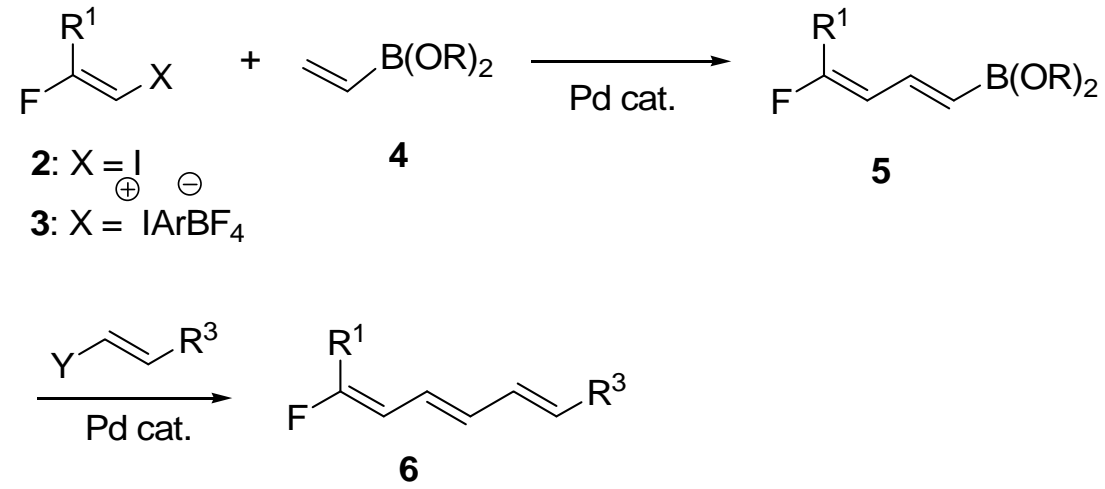

Scheme 2

\section{Result and discussion}

In the Heck-reaction of the vinylboronates with haloalkenes, the Suzuki-Miyaura coupling reaction competes to give dienes and the yield of the desired dienylboronates decreases [10]. Recently, Whiting et al. successfully depressed the undesired Suzuki-Miyaura coupling using 4,4,6-trimethyl-2-vinyl-1,3,2-dioxaborinane as the vinylboronate and performing the reaction at a high temperature [11]. However, under 
the same conditions, the Heck reaction of (E)-2-fluoro-1-iodoalkenes (2) with vinylboronates such as 4,4,6-trimethyl-2-vinyl-1,3,2-dioxaborinane or 4,4,5,5-tetramethyl-2-vinyl-[1,3,2]-dioxaborolane (7) proceeded non-selectively to give a mixture of products, e.g. (E)- and (Z)-fluorodienylboronates, and the Suzuki-Miyaura coupling product. Alkenyliodonium salts are more reactive than the corresponding iodides in the cross-coupling reaction $[15,16]$, and therefore, the Heck reaction of (E)-2-fluoro-1-dodecenyl iodonium salt (3a) with 4,4,5,5-tetramethyl-2-vinyl-[1,3,2]-dioxaborolane (7) was examined (Table 1). As expected, the reaction proceeded at room temperature and the desired (1E, 3E)-4-fluoro-1,3-tetradecadienylboronate (5a) was obtained stereoselectively (>95\%). Under those conditions, (2-tolylvinyl)boronate (9), the Heck reaction product with a tolyl group on 3a, and 4-fluoro-1,3-tetradecadiene (8), the Suzuki-Miyaura coupling product, were also formed as by-products [17]. As reported previously, amine is superior to $\mathrm{K}_{2} \mathrm{CO}_{3}$ as a base to selectively obtain the Heck product (entries 2 and 4) [18]. When the reaction was carried out using $\mathrm{Bu}_{3} \mathrm{~N}$ as a base and 2.4 eq of 7 to $3 \mathbf{a}, 5 \mathbf{a}$ was obtained in 65\% yield (entry 5). Under these conditions, 8 (5\% yield) and 9 (3\% yield), which can be separated from 5a by silica gel column chromatography, were also formed. 
Table 1

Synthesis of fluoroalkadienylboronate by Heck reaction using fluoroalkenyliodonium salt ${ }^{\mathrm{a}}$
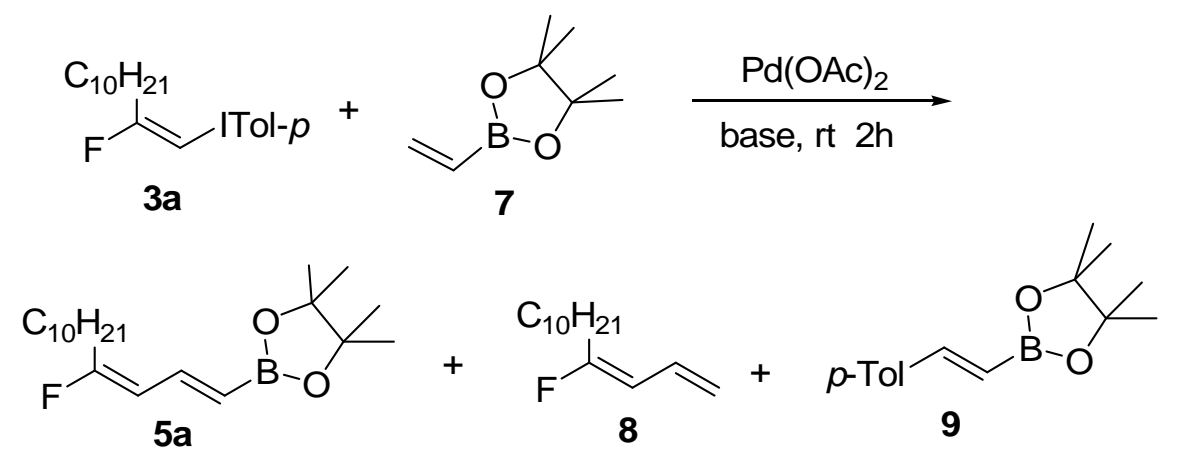

\begin{tabular}{cccccc}
\hline \multirow{2}{*}{ Entry } & Solvent & \multirow{2}{*}{ Base } & Yield of $\mathbf{5 a}(\%)^{\mathrm{b}}$ & \multicolumn{3}{c}{${\text { Yield of } \mathbf{8} \text { and } \mathbf{9}(\%)^{\mathrm{c}}}^{\mathbf{9}}$} & $\mathbf{9}$ \\
\hline 1 & toluene & $\mathrm{Bu}_{3} \mathrm{~N}$ & $40(97)$ & 2 & 3 \\
2 & $\mathrm{DMF}$ & $\mathrm{Bu}_{3} \mathrm{~N}$ & $60(96)$ & 10 & 4 \\
3 & $\mathrm{THF}$ & $\mathrm{Bu}_{3} \mathrm{~N}$ & $60(97)$ & 10 & 3 \\
4 & $\mathrm{DMF}$ & $\mathrm{K}_{2} \mathrm{CO}_{3}$ & $19(96)$ & 6 & 2 \\
5 & $\mathrm{DMF}$ & $\mathrm{Bu}_{3} \mathrm{~N}$ & $65(96)^{\mathrm{d}}$ & 5 & 3 \\
\hline
\end{tabular}

${ }^{a}$ If otherwise not mentioned, the reaction was carried out using $1.2 \mathrm{eq}$ of 7, 1.2 eq of base, and $5 \mathrm{~mol} \%$ of $\mathrm{Pd}(\mathrm{OAc})_{2}$.

${ }^{\mathrm{b}}$ Isolated yield base on $3 \mathbf{a}$. In parenthese, stereoselectivity, and a (1E, 3Z)-isomer was formed as by-product.

${ }^{\mathrm{C}}$ Determined by GC.

$\mathrm{d}_{2.4}$ eq of 7 was used.

Various 2-fluoroalkenyliodonium salts (3a-f) were used in the synthesis of 4-fluoro-1,3-alkadienylboronates 5a-f (Table 2). When (E)-fluoroalkenyliodonium salts (3a-e) were used, the corresponding (1E, 3E)-4-fluoro-1,3-alkadienylboronates (5a-e) were obtained in 55-65\% yield with good stereoselectivity (94-98\%). Functional groups such as ketone and ester can tolerate these reaction conditions, and functionalized fluoroalkadienylboronates (5c-e) were obtained (entries 3-5). On the other hand, the reaction with (Z)-fluoroalkenyliodonium salt (3f) proceeded less selectively, and the desired (1E, 3Z)-4-fluoro-1,3-alkadienylboronate (5f) was obtained 
in lower yield (46\%) with a significant amount of the Suzuki-Miyaura coupling product (20\%) (Entry 6) [17].

Table 2

Synthesis of fluoroalkadienylboronates by Heck reaction using various fluoroalkenyliodonium salt ${ }^{\mathrm{a}}$

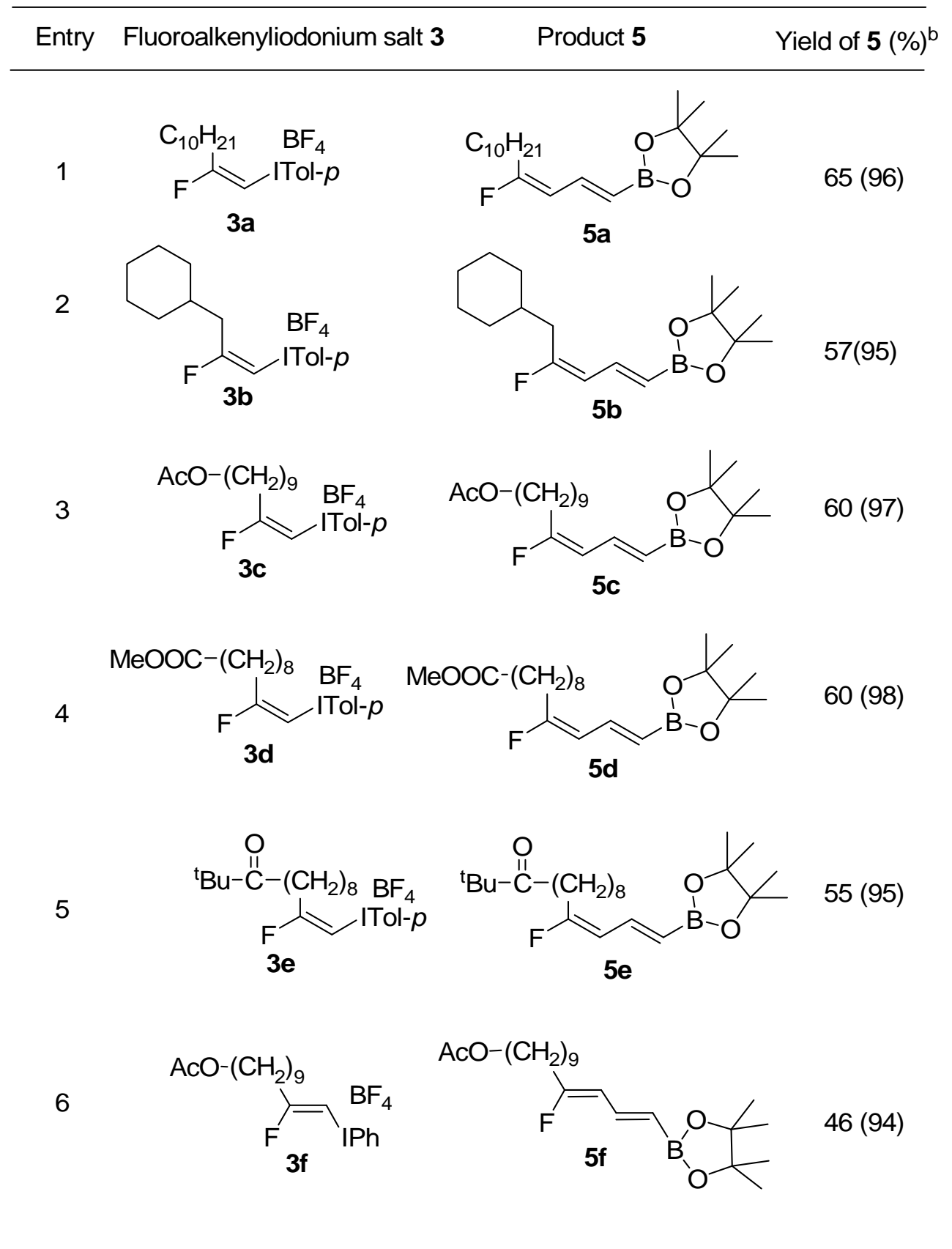

a If otherwise not mentioned, the reaction was carried out using $2.4 \mathrm{eq}$ of 7 , 1.2 eq of $\mathrm{Bu}_{3} \mathrm{~N}$, and $5 \mathrm{~mol} \%$ of $\mathrm{Pd}(\mathrm{OAc})_{2}$.

${ }^{\mathrm{b}}$ Isolated yield base on $\mathbf{3}$. In parenthese, stereoselectivity determined by ${ }^{19}$ FNMR. 
The resulting fluoroalkadienylboronates $\mathbf{5}$ can be used for fluoropolyene synthesis by the Suzuki-Miyaura coupling reaction. When 5a was subjected to the coupling reaction with ethyl 4-iodobenzoate (10), arylated (1E, 3E)-4-fluorotetradecadiene (12) was obtained in good yield. Similarly, (1E, 3E, 5E)-1-phenyl-6-fluorohexadecatriene (13) was obtained by the coupling reaction with $\beta$-bromostyrene (11) (Scheme 3).

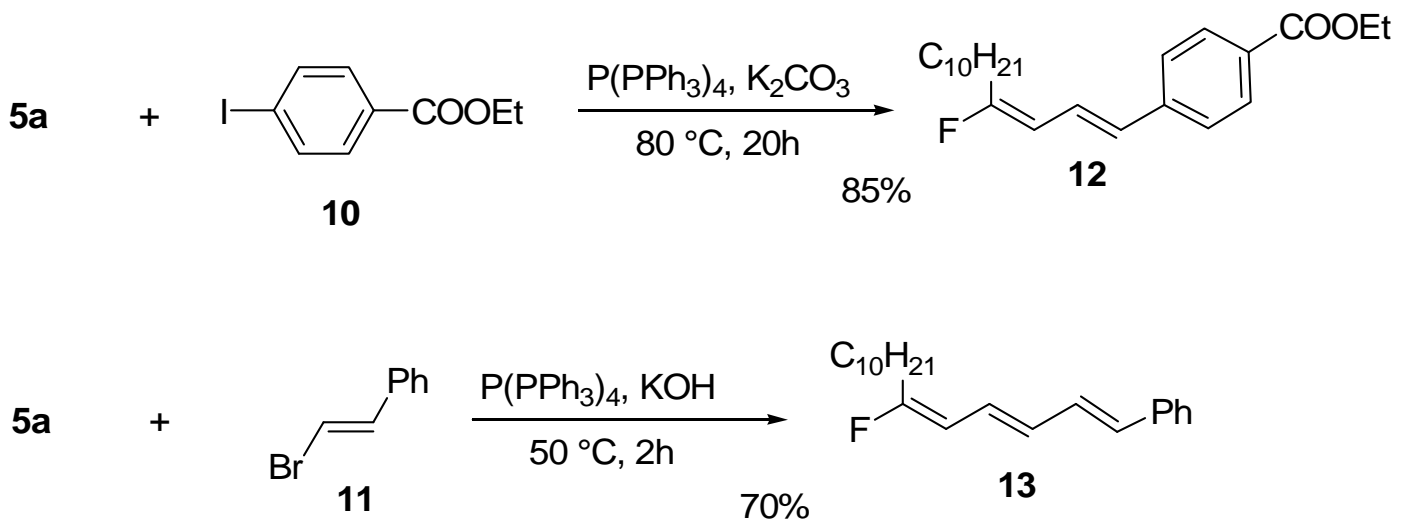

Scheme 3

\section{Conclusion}

In conclusion, various (1E, 3E)- and (1E, 3Z)-4-fluoro-1,3-alkadienylboronates were stereoselectively prepared

by

the

Heck-reaction

of 4,4,5,5-tetramethyl-2-vinyl-[1,3,2]-dioxaborolane

with

$(E)-$

or (Z)-2-fluoro-1-alkenyliodonium salts. The reaction proceeded at room temperature and the pure fluoroalkadienylboronates were isolated by column chromatography. The resulting fluoroalkadienylboronates can be used for the synthesis of fluoropolyenes by the Suzuki-Miyaura coupling reaction with aryl or alkenyl halides. 


\section{Experimental}

\subsection{General methods}

The IR spectra were recorded using a JASCO FT/IR-410 spectrophotometer. The ${ }^{1} \mathrm{H}$ NMR (400 MHz) spectra, ${ }^{19} \mathrm{~F}$ NMR (376 MHz) spectra and ${ }^{13} \mathrm{C}$ NMR (100 $\mathrm{MHz}$ ) spectra were recorded in $\mathrm{CDCl}_{3}$ on a JEOL JNM-A400II FT NMR spectrometer and the chemical shift, $\delta$, are referred to TMS $\left({ }^{1} \mathrm{H},{ }^{13} \mathrm{C}\right)$ and $\mathrm{CFCl}_{3}\left({ }^{19} \mathrm{~F}\right)$, respectively. The EI-high-resolution mass spectra were measured on a JEOL JMS-700TZ spectrometer.

(E)-2-Fluoroalkenyliodonium salts 3a-e were prepared from the corresponding alkynes and iodotoluene difluoride according to a literature [13]. (Z)-2-Fluoroalkenyliodonium salt 3f was prepared from the corresponding 1-alkyne in two steps according to the literatures [14]. 4,4,5,5-Tetramethyl-2-vinyl-[1,3,2]-dioxaborolane 7 was purchased from Aldrich or prepared according to the literature [19].

4.2. Synthesis of fluoroalkadienylboronates

\subsection{1.}

(1E,3E)-2-(4-Fluoro-1,3-tetradecadienyl)-4,4,5,5-tetramethyl-[1,3,2]-dioxaborolane

A mixture of $\mathrm{Pd}(\mathrm{OAc})_{2}(11 \mathrm{mg}, 0.05 \mathrm{mmol}), \mathrm{Bu}_{3} \mathrm{~N}$ (110 mg, $\left.0.6 \mathrm{mmol}\right)$, 3a (238 mg, $0.5 \mathrm{mmol}$ ), and 7 (185 mg, $1.2 \mathrm{mmol})$ in DMF (1.5 ml) was stirred under $\mathrm{N}_{2}$ atmosphere at room temperature for $2 \mathrm{~h}$. Then, the mixture was poured into water and extracted with ether three times. The combined organic layers were dried over $\mathrm{MgSO}_{4}$ and 
concentrated under reduced pressure. Purification by column chromatography (silica gel/hexane-ether) gave 5a (110 mg, $0.33 \mathrm{mmol}$ ) in 65\% yield. IR (neat) 2927, 1665, $1145 \mathrm{~cm}^{-1} . \quad{ }^{1} \mathrm{H}$ NMR $\delta 0.88(\mathrm{t}, J=6.8 \mathrm{~Hz}, 3 \mathrm{H}), 1.27(\mathrm{~s}, 12 \mathrm{H}), 1.27-1.31(\mathrm{~m}, 14 \mathrm{H})$, $1.52-1.54$ (m, 2H), 2.42 (dt, $J=23.4,7.3 \mathrm{~Hz}, 2 \mathrm{H}$ ), 5.48 (d, $J=17.5 \mathrm{~Hz}, 1 \mathrm{H}$ ), 5,82 (dd, $J=19.8,11.2 \mathrm{~Hz}, 1 \mathrm{H}), 6.97(\mathrm{dd}, J=17.5,11.3 \mathrm{~Hz}, 1 \mathrm{H}) .{ }^{19} \mathrm{~F}$ NMR $\delta-95.64(\mathrm{dt}, J=$ 19.6, 23.3 Hz, 1F). ${ }^{13} \mathrm{C}$ NMR $\delta 14.10,22.67,24.73$ (4C), 26,32, 28.64 (d, $J=25.9 \mathrm{~Hz}$ ), 28.98, 29.29, 29.31, 29.46, 29.55, 31.88, 83.15 (2C), 110.62 (d, $J=26.5$ Hz), 142.99 (d, $J=12.4 \mathrm{~Hz}$ ), 166.10 (d, $J=261.2 \mathrm{~Hz}$ ). $\mathrm{HRMS}(\mathrm{EI})$ : calc. for $\mathrm{C}_{20} \mathrm{H}_{36} \mathrm{O}_{2} \mathrm{FB}: 338.2792$, found 338.2794.

4.2.2. (1E,3E)-2-(5-Cyclohexyl-4-fluoro-1,3-pentadienyl)-4,4,5,5-tetramethyl-[1,3,2]dioxaborolane (5b). IR (neat) 2926, 1663, 1608, 1331, $1146 \mathrm{~cm}^{-1} . \quad{ }^{1} \mathrm{H}$ NMR $\delta 1.16$ - 1.26 (m, 10H), 1.28 (s, 12H), $1.64-1.72$ (m, 1H), 2.31 (dd, $J=24.5,7.1 \mathrm{~Hz}, 2 \mathrm{H}$ ), 5.47 (d, $J=17.5 \mathrm{~Hz}, 1 \mathrm{H}$ ), 5.87 (dd, $J=20.1,11.2 \mathrm{~Hz}, 1 \mathrm{H}$ ), 6.93 (dd, $J=17.5,11.2 \mathrm{~Hz}$, 1H). $\quad{ }^{19}$ F NMR $\delta$ - $93.15(\mathrm{dt}, J=20.2,24.4 \mathrm{~Hz}, 1 \mathrm{~F}) . \quad{ }^{13} \mathrm{C}$ NMR $\delta 24.73(4 \mathrm{C}), 26.13$ (2C), 26.25, 32.86 (2C), 35.40, 36.33 (d, $J=26.0 \mathrm{~Hz}$ ), 83.13 (2C), 111.73 (d, $J=26.6$ Hz), 143.00 (d, $J=12.1 \mathrm{~Hz}$ ), 164.99 (d, $J=261.2 \mathrm{~Hz}$ ). HRMS (EI): calc. for $\mathrm{C}_{17} \mathrm{H}_{28} \mathrm{O}_{2} \mathrm{FB}:$ 294.2166, found 294.2149.

4.2.3. (1E,3E)-2-(13-Acetoxy-4-fluoro-1,3-tridecadienyl)-4,4,5,5-tetramethyl-[1,3,2]dioxaborolane (5c). IR (neat) 2931, 1741, 1664, 1608, 1335, 1240, $1146 \mathrm{~cm}^{-1} .{ }^{1} \mathrm{H}$ NMR $\delta 1.27$ (s, 12H), 1.27 - 1.30 (m, 10H), 1.55 - 1.62 (m, 4H), 2.05 (s, 3H), 2.42 (dt, $J=23.4,7.6 \mathrm{~Hz}, 2 \mathrm{H}), 4.05$ (t, $J=6.7 \mathrm{~Hz}, 2 \mathrm{H}), 5.48$ (d, $J=17.5 \mathrm{~Hz}, 1 \mathrm{H}), 5.82$ (dd, $J=$ 19.8, 11.3 Hz, 1H), 6.96 (dd, $J=17.6,11.4 \mathrm{~Hz}, 1 \mathrm{H}) . \quad{ }^{19} \mathrm{~F}$ NMR $\delta$ - 95.70 (dt, $J=19.5$, 23.2 Hz, 1F). ${ }^{13} \mathrm{C}$ NMR $\delta$ 20.81, 24.59 (4C), 25.73, 26.14, 28.43. 28.50 (d, $J=26.2$ Hz), 28.75, 29.03, 29.09, 29.17, 64.45, 83.01 (2C), 110.53 (d, $J=26.2 \mathrm{~Hz}), 142.79$ (d, $J$ $=12.4 \mathrm{~Hz}$ ), $165.84(\mathrm{~d}, J=261.3 \mathrm{~Hz}), 171.04$. HRMS (EI): calc. for $\mathrm{C}_{21} \mathrm{H}_{36} \mathrm{O}_{4} \mathrm{FB}$ : 
382.2690, found 382.2681.

4.2.4. Methyl (10E,12E)-10-fluoro-13-(4,4,5,5-tetramethyl-[1,3,2]-dioxaborolan2-yl)-10, 12-tridecadienoate (5d). IR (neat) 2932, 1740, 1664, 1608, $1335 \mathrm{~cm}^{-1} \cdot{ }^{1} \mathrm{H}$ NMR $\delta 1.27$ (s, 12H), $1.27-1.31$ (m, 8H), $1.43-1.62$ (m, 4H), 2.30 (t, J = 7.6 Hz, 2H), 2.42 (dt, $J=23.4,7.6 \mathrm{~Hz}, 2 \mathrm{H}), 3.67$ (s, 3H), 5.48 (d, $J=17.6 \mathrm{~Hz}, 1 \mathrm{H}), 5.82$ (dd, $J=$ 19.7, 11.2 Hz, 1H), 6.96 (dd, $J=17.6,11.2 \mathrm{~Hz}, 1 \mathrm{H}) . \quad{ }^{19} \mathrm{~F}$ NMR $\delta$ - 95.71 (dt, $J=20.2$, $23.2 \mathrm{~Hz}, 1 \mathrm{~F}) .{ }^{13} \mathrm{C}$ NMR $\delta 24.63$ (4C), 24.77, 26.16, 28.54 (d, $\left.J=26.5 \mathrm{~Hz}\right), 28.75$, 28.94 (2C), 29.01, 33.92, 51.28, 83.03 (2C), 110.56 (d, $J=26.5 \mathrm{~Hz}), 142.80$ (d, $J=12.4$ Hz), 165.86 (d, $J=261.3 \mathrm{~Hz}$ ), 174.10. HRMS (EI): calc. for $\mathrm{C}_{20} \mathrm{H}_{34} \mathrm{O}_{4} \mathrm{FB}$ : 368.2534, found 368.2526.

4.2.5.

(12E,14E)-12-Fluoro-2,2-dimethyl-15-(4,4,5,5-tetramethyl-[1,3,2]-dioxaborolan-2-yl)12, 14-pentadecadien-3-one (5e). IR (neat) 2931, 1705, 1664, 1608, $1334 \mathrm{~cm}^{-1} .{ }^{1} \mathrm{H}$ NMR $\delta 1.13$ (s, 9H), 1.27 (s, 12H), 1.27 - 1.30 (m, 8H), 1.54 - 1.55 (m, 4H), 2.37 2.48 (m, 4H), 5.48 (d, $J=17.5 \mathrm{~Hz}, 1 \mathrm{H}), 5.82$ (dd, $J=19.8,11.2 \mathrm{~Hz}, 1 \mathrm{H}), 6.96$ (dd, $J=$ 17.6, 11.4 Hz, 1H). ${ }^{19} \mathrm{~F}$ NMR $\delta$ - 95.67 (dt, $\left.J=19.9,23.5 \mathrm{~Hz}, 1 \mathrm{~F}\right) . \quad{ }^{13} \mathrm{C}$ NMR $\delta 23.85$, 24.72 (4C), 26.28, 26.37 (3C), 28.66 (d, $J=26.5$ Hz), 28.91, 29.20, 29.22, 29.31, 36.35, 44.05, 83.13 (2C), 110.62 (d, $J=26.6 \mathrm{~Hz}), 142.93$ (d, $J=12.4 \mathrm{~Hz}), 166.02$ (d, $J=261.5$ $\mathrm{Hz}$ ), 216.08. HRMS (EI) calc. for $\mathrm{C}_{23} \mathrm{H}_{40} \mathrm{O}_{3} \mathrm{FB}$ : 394.3054, found 394.3056.

4.2.6. (1E,3Z)-2-(13-Acetoxy-4-fluoro-1,3-tridecadienyl)-4,4,5,5-tetramethyl[1,3,2]-dioxaborolane (5f). $\quad$ IR (neat) 2930, 1740, $1360 \mathrm{~cm}^{-1} . \quad{ }^{1} \mathrm{H}$ NMR $\delta 1.24-1.32$ (m, 10H), 1.27 (s, 12H), 1.51 - 1.63 (m, 4H), 2.05 (s, 3H), 2.21 (dt, $J=17.6,7.4 \mathrm{~Hz}$, 2H), 4.05 (t, $J=6.7 \mathrm{~Hz}, 2 \mathrm{H}), 5.35$ (dd, $J=35.0,10.9 \mathrm{~Hz}, 1 \mathrm{H}), 5.42$ (d, $J=18.1 \mathrm{~Hz}, 1 \mathrm{H}$ ), $7.29(\mathrm{dd}, J=17.9,10.9 \mathrm{~Hz}, 1 \mathrm{H}) . \quad{ }^{19} \mathrm{~F}$ NMR $\delta-98.26(\mathrm{dt}, J=36.4,17.7 \mathrm{~Hz}, 1 \mathrm{~F}) . \quad{ }^{13} \mathrm{C}$ 
NMR $\delta$ 20.98, 24.72 (4C), 25.85, 25.95, 28.55, 28.81, 29.15, 29.29 (2C), 32.21(d, $J=$ 25.7 Hz), 64.58 (2C), 83.11, 108.72 (d, $J=11.4 \mathrm{~Hz}), 141.42$ (d, $J=5.7 \mathrm{~Hz}), 163.14$ (d, $J=269.9 \mathrm{~Hz}), 171.19$.

\subsection{Suzuki-Miyaura reaction using $5 a$}

\subsubsection{Ethyl 4-\{(1E,3E)-4-fluoro-1,3-tetradecadienyl\}benzoate (12)}

A mixture of $\mathrm{Pd}\left(\mathrm{PPh}_{3}\right)_{4}(28.8 \mathrm{mg}, 0.025 \mathrm{mmol})$, ethyl p-iodobenzoate (166 mg, 0.6 mmol), 5 a (169 mg, $0.5 \mathrm{mmol})$, aq $\mathrm{K}_{2} \mathrm{CO}_{3}$ (0.6 ml of 2M solution), and $\mathrm{EtOH}$ (0.6 ml) in toluene (5 ml) was stirred under $\mathrm{N}_{2}$ atmosphere at $80{ }^{\circ} \mathrm{C}$ for $20 \mathrm{~h}$. The mixture was poured into water and extracted with ether three times. Combined organic layers were dried over $\mathrm{MgSO}_{4}$ and concentrated under reduced pressure. Purification by column chromatography (silica gel/hexane-ether) gave 12 (153 mg, $0.43 \mathrm{mmol}$ ) in 85\% yield. IR (neat) 2926, 2855, 1718, 1665, 1604, $1276 \mathrm{~cm}^{-1} . \quad{ }^{1} \mathrm{H}$ NMR $\delta 0.87$ (t, $J=7.1 \mathrm{~Hz}, 3 \mathrm{H}$ ), $1.26-1.43$ (m, 14H), 1.40 (t, $J=7.1 \mathrm{~Hz}, 3 \mathrm{H}), 1.55-1.61$ (m, 2H), 2.46 (dt, $J=23.4$, $7.4 \mathrm{~Hz}, 2 \mathrm{H}), 4.37$ (q, $J=7.1 \mathrm{~Hz}, 2 \mathrm{H}), 5.93$ (dd, $J=19.5,11.3 \mathrm{~Hz}, 1 \mathrm{H}), 6.49$ (d, $J=15.5$ Hz, 1H), 6.78 (dd, $J=15.1,11.5 \mathrm{~Hz}, 1 \mathrm{H}), 7.41$ (d, $J=8.4 \mathrm{~Hz}, 2 \mathrm{H}), 7.98$ (d, $J=8.3 \mathrm{~Hz}$,

2H). $\quad{ }^{19} \mathrm{~F}$ NMR $\delta$ - $96.81(\mathrm{dt}, J=19.9,23.1 \mathrm{~Hz}, 1 \mathrm{~F}) . \quad{ }^{13} \mathrm{C}$ NMR $\delta$ 14.01, 14.22, 22.59, 26.30, 28.62 (d, $J=26.8 \mathrm{~Hz}), 28.87,29.24,29.26,29.44,29.52,31.81,60.72,108.56$ (d, $J=28.4 \mathrm{~Hz}$ ), 124.53 (d, $J=11.2 \mathrm{~Hz}), 125.66$ (2C), 128.79, 129.58(d, $J=10.1 \mathrm{~Hz})$, 129.82 (2C), 141.72, 164.80 (d, $J=259.4 \mathrm{~Hz}$ ), 166.20. HRMS(EI) calc. for $\mathrm{C}_{23} \mathrm{H}_{33} \mathrm{FO}_{2}: 360.2464$, found: 360.2470.

\subsection{2. (1E, 3E, 5E)-6-Fluoro-1-phenyl-1,3,5-hexadecatriene (13)}

A mixture of $\mathrm{Pd}\left(\mathrm{PPh}_{3}\right)_{4}(28.8 \mathrm{mg}, 0.025 \mathrm{mmol}),(E)-\beta$-bromostyrene (165 mg, 0.9 
mmol), 5a (169 mg, $0.5 \mathrm{mmol})$, aq $\mathrm{KOH}(0.6 \mathrm{ml}$ of $2 \mathrm{M}$ solution), and $\mathrm{EtOH}(0.6 \mathrm{ml})$ in toluene (5 ml) was stirred under $\mathrm{N}_{2}$ atmosphere at $50{ }^{\circ} \mathrm{C}$ for $2 \mathrm{~h}$. The mixture was poured into water and extracted with ether three times. Combined organic layers were dried over $\mathrm{MgSO}_{4}$ and concentrated under reduced pressure. Purification by column chromatography (silica gel/hexane-ether) gave 13 (110 mg, $0.35 \mathrm{mmol})$ in 70\% yield. IR (neat) 2926, 2854, 1663, 1604, $984 \mathrm{~cm}^{-1} . \quad{ }^{1} \mathrm{H}$ NMR $\delta 0.87$ (t, $\left.J=6.5 \mathrm{~Hz}, 3 \mathrm{H}\right), 1.26$ (brs, 14H), $1.31-1.58$ (m, 2H), 2.39 (dt, $J=23.4,7.3 \mathrm{~Hz}, 2 \mathrm{H}), 5.84$ (dd, $J=19.6,10.1$ Hz, 1H), $6.22-6.35$ (m, 2H), 6.51 (d, $J=15.6 \mathrm{~Hz}, 1 \mathrm{H}$ ), 6.83 (dd, $J=15.4,9.5 \mathrm{~Hz}, 1 \mathrm{H}$ ), $7.21-7.40(\mathrm{~m}, 5 \mathrm{H}) . \quad{ }^{19} \mathrm{~F}$ NMR $\delta-98.91(\mathrm{dt}, J=19.9,23.1 \mathrm{~Hz}, 1 \mathrm{~F}) .{ }^{13} \mathrm{C}$ NMR $\delta$ 14.11, 22.68, 26.41, 28.65 (d, $J=27.1 \mathrm{~Hz}$ ), 28.98, 29.32, 29.33, 29.51, 29.58, 31.89, 108.69 (d, $J=28.2 \mathrm{~Hz}), 126.21(2 \mathrm{C}), 126.35$ (d, $J=10.8 \mathrm{~Hz}), 127.34,128.60$ (2C), 129.06, 131.44, 131.50 (d, $J=7.2 \mathrm{~Hz}), 137.39,163.81$ (d, $J=258.0 \mathrm{~Hz}) . \quad$ HRMS (EI): calc. for $\mathrm{C}_{22} \mathrm{H}_{31} \mathrm{~F}$ : 314.2410, found: 314.2415.

\section{References}

[1] C. Thirsk, A. Whiting, J. Chem. Soc., Perkin Trans 1 (2002) 999-1023.

[2] I. Kadota, Y. Hu, G. K. Packard, S. D. Rychnovsky, Proc. Natl. Acad. Sci. U.S.A. 101 (2004) 11992-11995.

[3] R. S. H. Liu, H. Matsumoto, A. E. Asato, M. Denny, Y. Shichida, T. Yoshizawa, F. W. Dahlquist, J. Am. Chem. Soc. 103 (1981) 7195-7201.

[4] A. E. Asato, A. Kini, M. Denny, R. S. H. Liu, J. Am. Chem. Soc. 105 (1983) 2923-2924.

[5] T. Shinada, N. Sekiya, N. Bojkova, K. Yoshihara, Synlett (1995) 1247-1248

[6] A. Francesch, R. Alvarez, S. López, A. R. de Lera, J. Org. Chem. 62 (1997) 310-319.

[7] L. U. Colmenares, X.-L. Zou, J. Liu, A. E. Asato, R. S. H. Liu, A. R. de Lera, R. Alvarez, J. Am. Chem. Soc. 121 (1999) 5803-5804.

[8] H. Tsuchikawa, N. Matsushita, N. Matsumori, M. Murata, T. Oishi, Tetrahedron Lett. 47 (2006) 6187-6191.

[9] “Cross-Coupling Reactions; N. Miyaura Ed. Springer; Berlin, 2002.

[10] S. K. Stewart, A. Whiting, Tetrahedron Lett. 36 (1995) 3925-3928. 
[11] G. N. Maw, C. Thirsk, J.-L. Toujas, M. Vaultier, A. Whiting, Synlett (2004) 1183-1186.

[12] S. Hara, M. Yoshida, T. Fukuhara, N. Yoneda, Chem. Commun. (1998) 965-966.

[13] M. Yoshida, K. Kawakami, S. Hara, Synthesis (2004) 2821-2824.

[14] M. Yoshida, S. Hara, Org. Lett. 5 (2003) 573-574.

[15] S.-K. Kang, H.-W. Lee, S.-B. Jang, P.-S. Ho, J. Org. Chem. 61 (1996) 4720-4724.

[16] S. K. Kang, T. Yamaguchi, T.-H. Kim, P.-S. Ho, J. Org. Chem. 61 (1996) 9082-9083.

[17] M. Yoshida, A. Komata, S. Hara, Tetrahedron 62 (2006) 8636-8645.

[18] A. P. Lightfoot, S. J. R. Twiddle, A. Whiting, Synlett (2006) 529-531.

[19] R. H. Wallace, K. K. Zong, Tetrahedron Lett. 33 (1992) 6941-6944. 